American Journal of Applied Sciences 5 (12): 1701-1706, 2008

ISSN 1546-9239

(C) 2008 Science Publications

\title{
Comparison between Brain Tissue Gray and White Matters in Tension Including Necking Phenomenon
}

\author{
${ }^{1}$ Sina Mehdizadeh, ${ }^{1}$ Mehdi Khoshgoftar, ${ }^{1}$ Siamak Najarian, ${ }^{2}$ Farhad Farmanzad and \\ ${ }^{1}$ Seyyed Amir Hooshiar Ahmadi \\ ${ }^{1}$ Department of Biomechanics, Faculty of Biomedical Engineering, \\ Amirkabir University of Technology, Tehran, Iran \\ ${ }^{2}$ Department of Biomechanics, Faculty of Mechanical Engineering, Iran University of Science and \\ Technology, Tehran, Iran
}

\begin{abstract}
Characterizing the differences between the mechanical properties of brain tissue gray and white matters is of importance in biomechanics of brain tissue and may find a variety of different applications in medicine. In this study, a comparison has been made between mechanical behavior of bovine brain tissue white and gray matters. Through a linear elastic theory and using Bridgman method, necking phenomenon is considered for brain tissue in tension test. Results show that gray and white matters have different mechanical properties and differences between true and nominal values (the effect of cross section changes of the samples during the test) are not negligible. Besides, it is shown that for certain strains, linear elastic theory is acceptable for brain tissue modeling. These results are in agreement with the literature.
\end{abstract}

Keywords: Brain Tissue, Mechanical Behavior, Bridgman Method

\section{INTRODUCTION}

Describing mechanical behavior of brain tissue is one of the most challenging and complicated issues in biomechanics. Mechanical modeling of brain tissue is important because it has a substantial number of applications in robotic surgery, surgeon training systems, and traumatic brain injury simulation as well as in modeling of hydrocephalus and designing of helmets ${ }^{[1-3]}$. Until 1970, only a few papers were published on mechanical properties of the brain tissue ${ }^{[4]}$, but just recently several groups have focused on structural properties of the brain tissue and different biomechanical models of brain tissue have been proposed.

Determining mechanical properties of brain tissue have been investigated through conducting tension ${ }^{[5]}$, compression ${ }^{[6-7]}$ and shear experiments usually on animal brain tissues using linear and/or nonlinear elastic, hyperelastic poroelastic or viscoelastic models ${ }^{[8-11]}$. To mention some examples, a linear viscoelastic model was proposed for brain tissue in a research that could explain the behavior of the brain tissue in lower strain rates in compression. In that study, experiments were conducted on swine brain tissue ${ }^{[12]}$. Also, in a recent study, a biphasic model based on experiments on human brain was proposed for brain tissue ${ }^{[13]}$.

Brain tissue white and gray matters are complex materials. Gray matter of the cerebral hemispheres consists of a mixture of neuronal cell bodies, their unmyelinated processes and neurogilia. White matter, found in subcortical regions, consists of myelinated axonal fibers surrounded by supporting cells (oligodendrocytes, astrocytes, ependyma and microglia) and blood vessels ${ }^{[14]}$. Characterizing the differences between the mechanical properties of brain tissue gray and white matters is of importance in biomechanics. In a study, shear modulus of white matter of corona radiate region and thalamus gray matter of human brain were compared ${ }^{[15]}$. Another study showed that white matter of bovine brain tissue is approximately 3 times greater than gray matter in shear ${ }^{[16]}$.

All materials in tension test, after a meaningful time from beginning of the experiment, undergo necking phenomenon, but in tension mode of brain tissue, owing to the flexibility of tissue, necking starts from beginning of the experiment. Since necking disrupts steady manner of uniaxial stress, standard equation of 
stress and strain cannot be applied. In view of this, equations for post-necking have been created ${ }^{[17-18]}$. It seems that in performing tension test on brain samples, cross section changes of the samples during the test would not be negligible and will affect the results. Since accurate models are needed for various applications introduced for mechanical modeling of brain tissue, it is worthwhile to consider cross section changes of the samples during the tests.

In current study, a comparison has been made between mechanical behavior of bovine brain tissue white and gray matters. For the first time, through a linear elastic theory with Bridgman method, necking phenomenon is considered for brain tissue in tension test. We use a picture analyzing approach through a computer program to trace cross section changes during the test.

\section{MATERIALS AND METHODS}

Linear Elastic Model with Considering Cross Section Change: In tension of brain tissue, owing to flexibility of tissue, necking starts from the beginning of the experiment. Since necking disrupt steady manner of uniaxial stress, standard equation of stress and strain cannot be applied and to obtain more accurate results, it is necessary to consider changes in cross section directly in the formulation. Here, a linear elastic theory with Bridgman method in tension has been applied for brain tissue.

To consider cross section changes in theory, true strain can be defined as ${ }^{[17-18]}$ :

$$
\varepsilon=\int_{0}^{\varepsilon} d \varepsilon=\int_{L_{0}}^{L_{f}} \frac{d L}{L}=\ln \frac{L_{0}}{L_{f}}
$$

where $L_{0}$ is the initial length and $L_{f}$ is the ultimate length of the sample. For true strain we have:

$$
\varepsilon=\ln (1+e)
$$

which $e$ is the engineering strain.

To obtain true stresses in tension, the Bridgman method can be used. For a cylindrical beam, Bridgman hypothesized the followings (Fig 1):
1. Strain distribution in minimum area is uniform.

2. Beam's longitudinal gridline in necking zone changes to a curve with radius of curvature of $1 / \rho$ :

$$
\frac{1}{\rho}=\frac{r}{a R}
$$

3. Ratio of principal stresses remains constant.

As per the Bridgman method, the equivalent uniaxial stress (the Bridgman stress) can be defined as a nominal stress $\left(\sigma_{a}\right)_{a v}$ corrected with a coefficient $k$ as follows:

$$
\sigma_{e}=\frac{\left(\sigma_{a}\right)_{a v}}{\left(1+\frac{2 R}{a}\right) \ln \left(1+\frac{a}{2 R}\right)}=k\left(\sigma_{a}\right)_{a v}
$$

where $k$ is:

$k=\left[\left(1+\frac{2 R}{a}\right) \ln \left(1+\frac{a}{2 R}\right)\right]^{-1}$

Finally, average stress can be defined as:

$$
\sigma_{\text {ave }}=1 / 2\left(\sigma_{i n i}+\sigma_{e}\right)
$$

where $\sigma_{i n i}$ is the stress at sample' initial cross section.

For analyzing the pictures to trace cross section changes during the test, we prepared a computer program (we named it the Brain Test) written in visual FoxPro. For each experiment, 10 pictures were taken to compute their geometrical information. First, a picture was imported to the program. In each process, for a picture, the scale pixels were converted to 10 millimeter length (scale's length). For tension test, both the diameter of the sample $a$ and the radius of the curvature $\rho$ at necking zone were calculated. For computing the radius of curvature, a circle was drawn along the curvature of the sample with applying 3 points. The program running can be seen in Fig. 2 . 


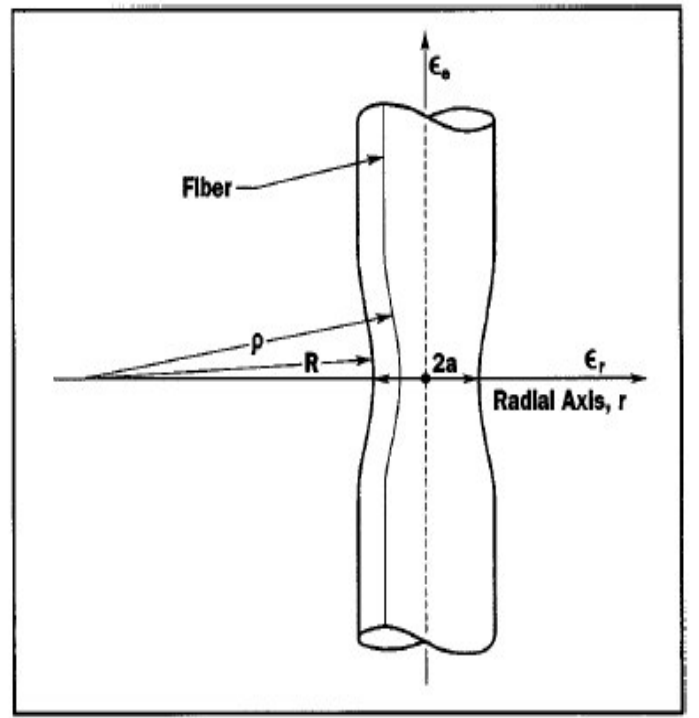

Fig 1: Schematic view of necking zone ${ }^{[18]}$.

Experiment: Experiments were conducted on gray and white matter of bovine brain tissue. Gray matter samples were harvested from parietal lobe and white matter samples were harvested from corpus callosum of a one year old bovine. In the case of white matter, cutting was perpendicular to axonal fibers alignment. Disk shape samples were $15 \mathrm{~mm}$ in diameter and $5 \mathrm{~mm}$ in height. Surgical scalpel was applied for cutting the samples. Experiments were conducted in less than 3 hours post-mortem. Before the start of the experiments, samples were preserved in physiological saline. No signs of dehydration of samples were observed during the experiments. Experiments were conducted at room temperature. The setup which was applied in this study had $0.01 \mathrm{~N}$ and 0.001 millimeter axial force resolution (Dynamic Testing Machine, Hct/25-400, Servo Hydraulic Valve PID Controller, Zwick/Roell Co., Germany) that uses Toolkit1998 software to acquire data (Fig. 3). Constant displacement was applied in the experiments for loading. Loading for all running was displacement with the rate of 1 $\mathrm{mm} / \mathrm{min}$. This amount of strain rate is convenient not to produce inertia forces on the sample ${ }^{[19]}$.

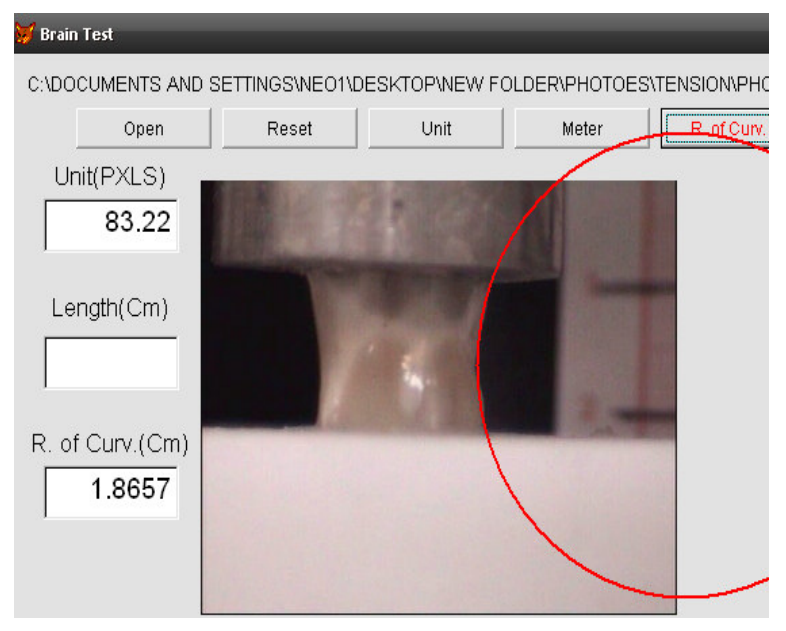

Fig 2: Brain Test program running, written in visual FoxPro for analyzing the pictures.

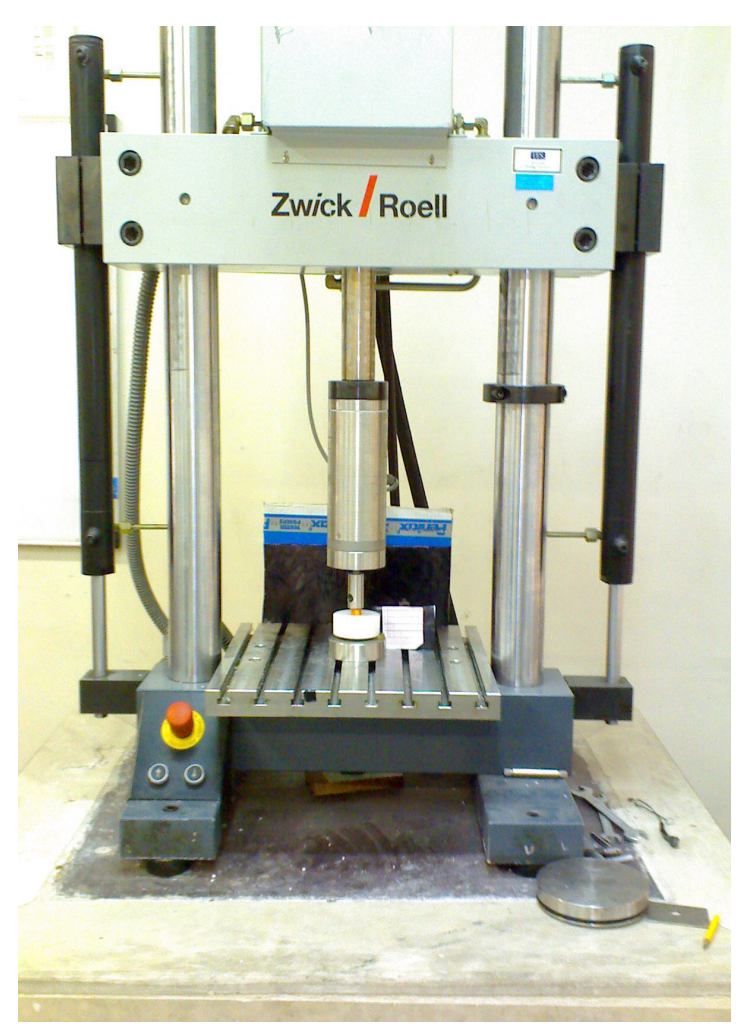

Fig 3: Experimental setup: brain sample is under tension and necking phenomenon has been accrued. 
In tension test, in order to prevent primary stress in the sample and subsequently error in the results, clamps cannot be used for placing of the samples in experimental setup. It has been proposed to use surgical adhesive to stick the sample to the setup. This method is suitable for minimizing the friction between sample and surface of the setup ${ }^{[11]}$. In this study, the upper and lower surfaces of the sample were stuck rigidly to the setup using a surgical adhesive. During experiments, there was not any slippage of the sample. Besides, due to the nature of methods used in this study (Bridgman method in tension), it was needed to know the change of the geometry of the samples during the test. Recording was done using a CCD camera. In order to analyze the pictures, they were taken on a black background.

\section{RESULTS AND DISCUSSION}

After conducting the experiments, loaddisplacement curves obtained from setup data were converted to the true stress-strain curves. For each experiment, two curves were plotted. Segmented line indicates the nominal stress-strain (load divided by initial cross section) and continued line is the average stress-strain curve calculated from Equation (6) for tension.

Figure 4 shows that sample cross section decreases with time and therefore true curves have a higher slope than the nominal one. This means that the true Young's modulus is greater than the nominal Young's modulus. As can be seen in Fig. 4, another issue which can be extracting out of the results is that for strains lower than $10 \%$, considering a linear elastic theory is acceptable for modeling the brain tissue gray matter. This conclusion is in agreement with the literature. In the case of brain tissue white matter, results show a unique behavior for strains lower than $10 \%$. For confirmation, we repeated our test and the same results derived. This unique behavior could be because of the fact that at the beginning of the test, axonal fibers of white matter are not in their original length (the end-to-end distance shown in Fig. 5) and consequently, their load bearing is a time-dependent phenomenon which leads to the mentioned unique behavior. For strains which fall in the range of $10 \%$ to $30 \%$, it is shown that linear elastic theory is acceptable for modeling the brain tissue white matter. Via drawing a tangent line from the base point of the curves related to gray matter and from the point in which the strain is $10 \%$ for curves related to white matter, Young modulus can be calculated.

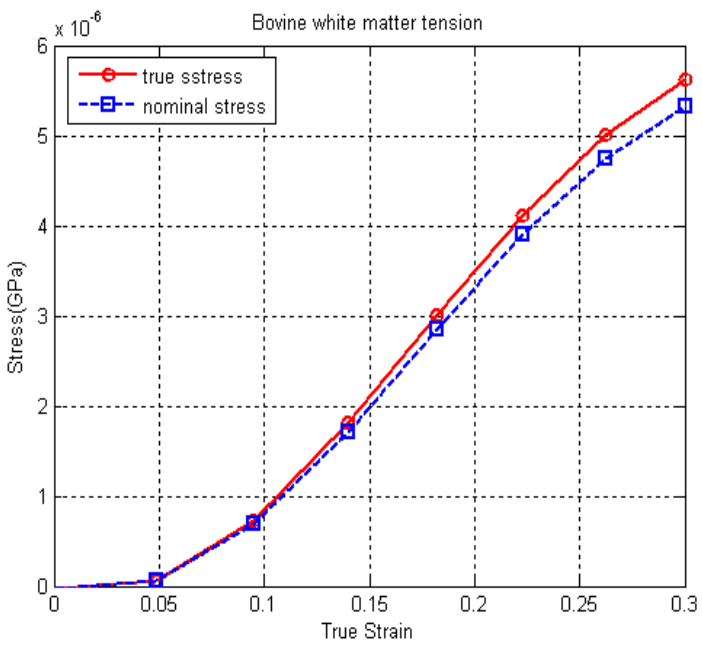

(a)

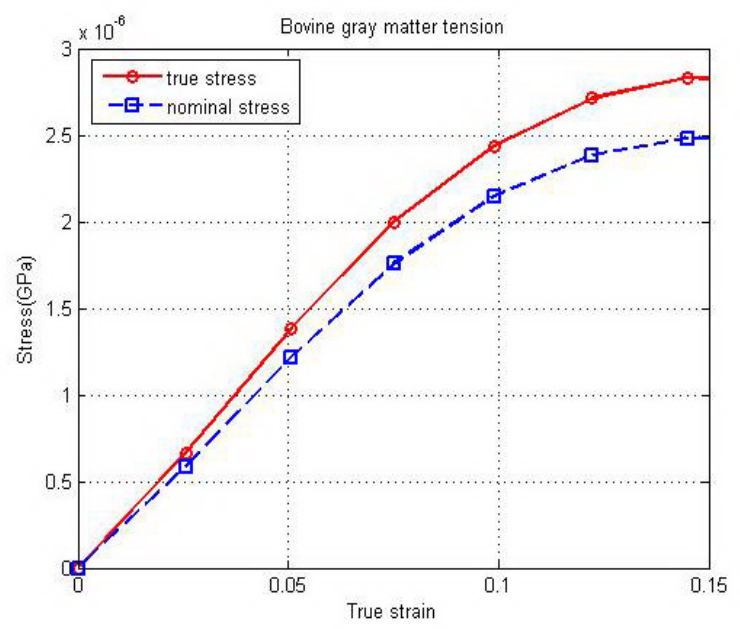

(b)

Fig 4: Stress-strain curves for (a) white matter and (b) gray matter.

Error in nominal values of Young modulus in comparison with true values of Young modulus for gray and white matters has been gathered in Table 1 .

Table 1: Error in nominal values of Young modulus in comparison with true values of Young modulus for gray and white matters

\begin{tabular}{clcc}
\hline & $\begin{array}{l}\text { Error in } \\
\text { modulus }(\%)\end{array}$ & nominal & Young \\
Test & Gray & White & \\
(Tension) & 11.7 & 5.2 & \\
\hline
\end{tabular}



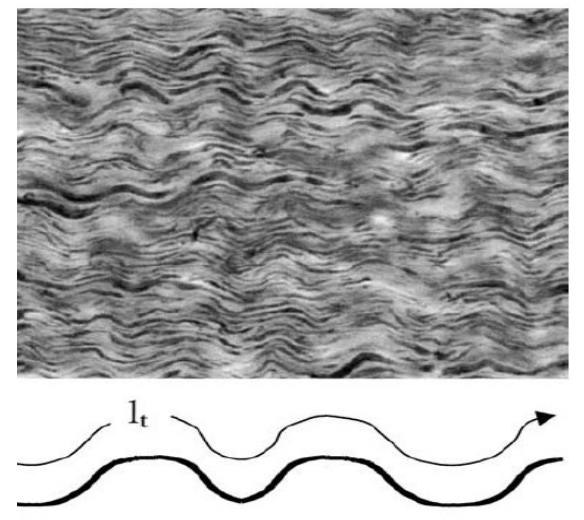

$1_{\mathrm{o}}$

University of Technology for their financial supports of this project.

\section{REFERENCES}

1. Farmanzad, F., S. Najarian, M. Eslami, A.S. Seddighi, 2007. A novel model for biomechanical behavior of human brain in epidural hematoma injuries, Bio-Medical Materials and Engineering, 17 (2): 119-125.

2. Pott, P.P., H-P. Scharf, M.L.R. Schwarz, 2005. Today's state of the art surgical robotics, Journal of Computer Aided Surgery, 10 (2): 101-132.

3. Taylor, Z., K. Miller, 2005. Reassessment of brain elasticity for analysis of biomechanisms of hydrocephalus, Journal of Biomechanical Engineering, 37: 1263-1269.

Fig 5: Microstructural geometry of myelinated Central Nervous System (CNS) axons within a white matter tract, $l_{t}$ is true axonal length and $l_{o}$ is the end-to-end distance ${ }^{[21]}$.

Mean difference between nominal values and true values of Young modulus (considering both gray and white matter) is $8.4 \%$. This amount of difference (which is because of unavoidable necking in tension) is substantial and it seems it should be considered in brain modeling. For gray matter, true Young modulus of 24.6 $\mathrm{kPa}$ and for white matter, true Young modulus of 19 $\mathrm{kPa}$ has been derived in our study. Theses results are close to the values introduced in the literature for brain tissue $^{[1,20]}$.

\section{CONCLUSION}

In this study, a comparison has been made between mechanical behavior of bovine brain tissue white and gray matters. Through a linear elastic theory with Bridgman method, necking phenomenon is considered for brain tissue in tension test. Results showed that gray and white matters have different mechanical properties. Besides, differences between true and nominal values (the effect of cross section changes of the samples during the test) are not negligible. Furthermore, it is shown that for certain strains, linear elastic theory is acceptable for brain tissue modeling. This result is in agreement with the literature.

\section{ACKNOWLEDGEMENTS}

The authors sincerely acknowledge the Center of Excellence in Biomedical Engineering of Iran located at the Faculty of Biomedical Engineering of Amirkabir

4. Ommaya, A.K., 1968. Mechanical properties of tissues of the nervous system, Journal of Biomechanics, 1: 127.

5. Miller, K., K. Chinzei, 2002. Mechanical properties of brain tissue in tension, Journal of Biomechanics, 35: 483.490.

6. Estes, M.S., J.H. McElhaney, 1970. Response of brain tissue of compressive loading, An ASME Publication, 70-BHF-13, 4.

7. Miller, K., 1997. Constitutive modeling of brain tissue: Experiment and theory, Journal of Biomechanics, 30: 1115-1121.

8. Bilston, L.E., Z. Liu, N. Phan-Thien, 2001. Large strain behavior of brain tissue in shear: Some experimental data and differential constitutive model, Biorheology, 38: 335-345.

9. Brands, D.W.A., P.H.M. Bovendeerd, G.W.M. Peters, J.S.H.M. Wismans, 2000. The large shear strain dynamic behavior of in-vitro porcine brain tissue and the silicone gel model material, Proceeding. of the 44th Stapp Car Crash Conference, 2000-01-SC17: 249-260.

10. Donnely, B.R., J. Medige, 1997. Shear properties of human brain tissue, Journal of Biomechanical Engineering, 119: 423-432.

11. Prange, M.T., S.S. Margulies, 2002. Regional, directional, and age-dependent properties of the brain undergoing large deformation, Journal of Biomechanical Engineering, 124: 244-252.

12. Miller, K., 1999. Constitutive model of brain tissue suitable for finite element analysis of surgical procedures, Journal of Biomechanics, 32: 531-537.

13. Franceschini, G., D. Bigoni, P. Regitni, G.A. Holzapfel, 2006. Brain tissue deforms similarly to filled elastomers and follows consolidation theory, J. of the Mechanics and Physics of Solids, 54: 2592-2620. 
14. Gefen, A., S. Margulies, 2004. Are in vivo and in situ brain tissues mechanically similar?, Journal of Biomechanics, 37: 1339-1352.

15. Shuck, L.Z. S.H. Advani, 1972. Rheological response of human brain tissue in shear, Journal of Basic Engineering, 94: 905-911.

16. Darvish, K.K., J.R. Crandall, 2001. Nonlinear viscoelastic effects in oscillatory shear deformation of brain tissue, Mechanical Engineering \& Physics, $23,633-645$.

17. Hashemi, J., R. Bennettv, 2003. Materials Characterization: Tensile Test, Material And Mechanics Laboratory, ME 3328.

18. Ling, Y., 1996. Uniaxial True Stress-Strain after Necking, AMP Journal of Technology, 5.
19. Velardi, F., F. Fraternali, M. Angelillo, 2006. Anisotropic constitutive equations and experimental tensile behavior of brain tissue, Biomechanics and Modeling in Mechanobiology, 5: 53-61.

20. Khoshgoftar, M., S. Najarian, F. Farmanzad, 2007. A Biomechanical Composite Model to Determine Effective Elastic Moduli of the CNS Gray Matter, American Journal of Applied Science, 4: 918-924.

21. Meaney, D. F., 2003. Relationship between structural modeling and hyperelastic material behavior: application to CNS white matter, Biomechanics and Modeling in Mechanobiology, 1: $279-293$. 